\title{
Recent Advances on Fluorescence Imaging of Lymphatic Vasculature System
}

\author{
Yi Li ${ }^{a}$ and Liqin Xiong ${ }^{*}, b$ \\ ${ }^{a}$ School of Medicine, Shanghai Jiao Tong University, Shanghai 200025, China \\ ${ }^{b}$ Shanghai Med-X Engineering Center for Medical Equipment and Technology, School of Biomedical \\ Engineering, Shanghai Jiao Tong University, Shanghai 200030, China
}

Email: xiongliqin@sjtu.edu.cn (L. X.)

\begin{abstract}
Lymphatic vasculature system has attracted extensive attention due to its important role in some pathological changes such as lymphedema, immune dysfunction, gut malabsorption and lymph node metastasis, etc. With the continuous development of fluorescence imaging technology, it has been widely used in the study of lymphatic vessels structure, morphology, and function both in preclinical and clinical. Fluorescence imaging can help researchers and clinicians to know the morphological and functional status of lymphatic vessels, explore the role of lymphatic vessels in various lymph-related diseases, and help diagnosis the state of lymphatic vessels in patients and formulate further treatment plan. Herein, we describe the usage and recent advances of fluorescence imaging technology including immune fluorescence imaging, optical coherence tomography, near-infrared fluorescence imaging in ex vivo and in vivo lymphangiography, and discuss the future applications of fluorescence imaging in lymphatic vasculature imaging.
\end{abstract}

Keywords lymphatic vasculature system, fluorescence imaging, lymphangiography

\section{Introduction}

A deeper understanding of the structure and function of lymphatic vasculature system helps the researchers further to understand the important role of the lymphatic system in the development of disease. Lymphatic vasculature system is associated with lymphedema, immune dysfunction, gut malabsorption, and so on, because it not only participates in tissue fluid homeostasis and immune trafficking, but also carries lipids from the gut to the blood circulation. ${ }^{[1-3]}$ Moreover, lymphatic vasculature system has relevance with cancer progression as the metastatic tumor cells can spread through the lymphatics into the lymph nodes. ${ }^{[3-7]}$ Imaging the lymphatic vasculature system is key to understand the role of lymphatic vessels in physiological and pathological process and design targeted therapeutic methods, and noninvasive imaging is indispensable for studying the relationship between lymphatic structure and function changes in disease development.

Fluorescence imaging has already become a widely used imaging techniques in biomedical research and clinical practice, which can image the structural and functional changes in living organism. ${ }^{[8-13]}$ With the discovery of specific biomarkers of lymphatic vessels, the distribution of lymphatic vessels in various tissues and its role in pathological progression is increasingly clear through immunofluorescence imaging. High-resolution imaging techniques including optical coherence tomography and light sheet microscopy let researchers further construct three-dimensional images of lymphatic vasculature system in each organ and provide more accurate and detailed morphological information such as structure, diameter and flow of lymphatic vessels. The development of in vivo fluorescence imaging techniques such as near-infrared fluorescence imaging further advances the non-invasive study of lymphatic vessels structure and function, and it has excellent clinical transformation value to help the clinicians diagnose the status of patients' lymphatic vessels and design the treatment progress.

In this paper, we firstly summarize the latest applications of fluorescence imaging techniques in lymphatic system ex vivo imaging, then introduce the development of lymphatic vasculature system fluorescence in vivo imaging techniques, and finally make a preliminary discussion and outlook of the future applications of fluorescence imaging techniques in lymphatic vasculature system imaging (Figure 1)

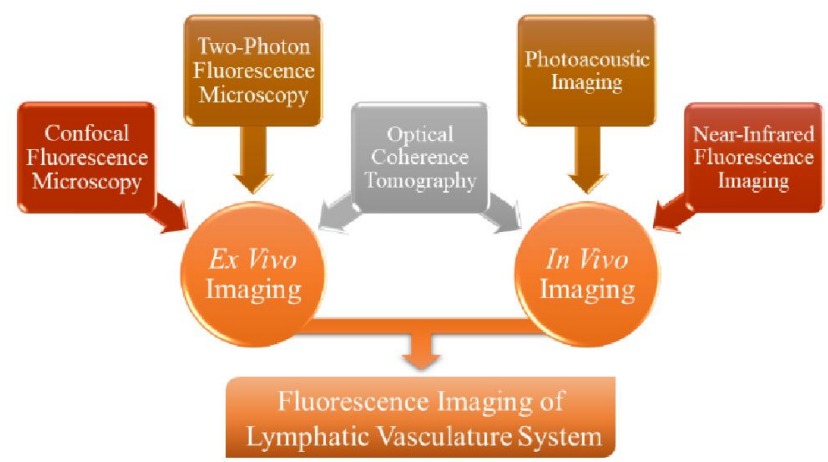

Figure 1 Summary of ex vivo and in vivo imaging techniques applied in lymphoangiography.

\section{Immunofluorescence Imaging and Confocal Fluorescence Microscopy}

Immunofluorescence imaging is a fluorescence imaging technique based on immunology, biochemistry and microscopic imaging technology. It firstly marks a known antigen or antibody with fluorophore and then uses the fluorescing antigen or antibody to target the corresponding antigen in cells and tissues according to the antigen-antibody reaction principle, thereby determining the nature and location of antigen or antibody in cells and tissues. The finding of lymphatic specific biomarkers promotes the research of lymphatic development, structure and function, ${ }^{[14,15]}$ and further advances the cognition of the role of lymphatic vessels in the process of disease progression. The commonly used biomarkers in lymphatic immunofluorescence 


\section{Minireview}

imaging are vascular endothelial growth factor receptor 3(VEGFR3), ${ }^{[16]}$ podoplanin, ${ }^{[17]}$ Prox-1, lymphatic vessel endothelial hyaluronan receptor 1 (LYVE-1), ${ }^{[14]} \mathrm{D} 2-40,{ }^{[17]} \mathrm{CD} 31,{ }^{[18]}$ etc The discovery of lymphatic-specific biomarkers has facilitated the study of the morphology of lymphatic vessels, and it has become a useful tool for researchers to understand the structure and function of lymphatic vessels.

Crohn's disease is a chronic inflammatory disease of the intestinal tract, and the pathological studies suggest that patients with Crohn's disease may have potential defects in their lymphatic transport. In mouse models, poor healing caused by infection-induced tissue damage may result in high permeability of the collecting lymphatic vessels in the mesenteric adipose tissue, and antigens and immune cells can thereby enter the mesenteric lymph nodes to maintain the appropriate level of immunity. ${ }^{[19-21]}$ Rehal et al. used immunofluorescence and confocal imaging techniques to acquire morphological and functional information of mesenteric lymphatic vessels in mice models with Crohn's disease. In this study, researchers found that the mouse model had same lymphatic abnormalities as human Crohn's disease including intestinal lymphangiectasia, mesenteric lymph node lymphadenopathy and lymphangiogenesis in mesentery and mesenteric mucosa, and mesenteric tertiary lymphoid organs also existed in mice models and alter the transportation of dendritic cells to the mesenteric lymph nodes, which may actively regulate immune responses. This study elucidated the effects of lymphatic vessels' changes found in Crohn's disease on immunity and suggested that the lymphatic system is a new target for the treatment of Crohn's disease. ${ }^{[22]}$ In order to find out whether pathological changes in the mouse model of Crohn's disease will occur in human intestinal diseases, Randolph et al. established a three-dimensional (3-D) imaging method, which used immunofluorescence and confocal imaging technique to describe the condition of the lymphatic vasculature system in the mesenteric tissue of patients with Crohn's disease and the control group. Through three-dimensional imaging, the researchers determined that there are tertiary lymphoid organs, which are known to exist in many chronic inflammatory diseases, in the mesenteric tissue of patients with Crohn's disease. The tertiary lymphoid organ changes the path of collecting lymphatic vessels into and out of the lymph nodes, thereby changing the flow of lymph in the mesenteric tissue. ${ }^{[23]}$

Lohrberg et al. decalcified the heads of 14-day-old mice, embedded the decalcified tissue in paraffin, stained with the lymph endothelial-specific antibodies LYVE-1 and podoplanin and imaged them to describe the lymphatic vasculature system in the head of the mouse. The findings discuss the drainage of cerebro-spinal fluid and the underlying pathways of ocular tumor transmission. ${ }^{[24]}$

Nizamutdinova et al. visualized lymphatic endothelial cell junctions by staining CD31 on lymphatic vessels to depict the location of lymphatic endothelial cells and lymphatic valves. To further elucidate the mechanism of aging-related effects on lymphatic contraction and to obtain the expression of histidine decarboxylase (HDC) in adult and elderly mesenteric lymphatic vessels (MLVs), the researchers used confocal microscopy to map the lymphatic vessels by referring the location of lymphatic endothelial cells which is stained by CD31. The lymphatic vessels were imaged and the immunohistochemical labeling results of histamine producing enzyme, HDC in adult and elderly MLVs were compared. They found increased expression of HDC in endothelium of aged MLVs. The results also provide the basis for further understanding the processes of aging lymphatic changes and new research directions for the agingassociated influence in lymph flow and immune response. ${ }^{[25]}$

\section{Two-Photon Fluorescence Microscopy}

Lymphocyte recirculation through lymph nodes requires chemoattractant-triggered cell migration to cross the endothelial barrier in blood and lymphatic vessels. However, the details of the migration of many lymphocytes across the endothelial barriers are still poorly understood. Park et al. injected Evans Blue solution, fluorescently labeled antibody of high endothelial venules (HEVs) and LYVE-1 into mice and used in vivo two-photon imaging techniques to visualize micro blood vessels, HEVs and lymphatic vessels. Through intravital two-photon imaging, researchers realized real-time observation of immune cells in the lymph nodes of the mouse and monitored the interactions between circulating lymphocytes and the stromal barriers. ${ }^{[26]}$

\section{Light-Sheet Fluorescence Microscopy}

When using thin sections for histological studies, the images are limited in providing 3-D structural information, particularly of the sophisticated morphological information of the vasculature. If thick samples imaging can overcome the limitations of low light penetration, high spatial resolution 3-D imaging of thick samples can be realized. Oren et al. designed the whole body vascular and lymphatic imaging (WOBLI) method for high-resolution 3-D imaging of blood vessels and lymphatic vessels in the ovary, uterus, lung, and liver of immunofluorescence labeling mice. The WOBLI imaging method includes immunolabeling, hydrogel embedding, tissue clearing and imaging, and the images of blood and lymphatic vessels obtained by light-sheet microscopy are constructed into complete 3-D structure images of whole organs (Figure 2). Combined with numerical analysis, the structural and functional status of blood and lymphatic vessels in various organs can be accurately evaluated, and 2-D image analysis cannot realize these high precision assessments. The WOBLI imaging protocol provides a reliable method for high-resolution 3-D imaging of wholeorgan blood vessels and lymphatic vessels. ${ }^{[27]}$
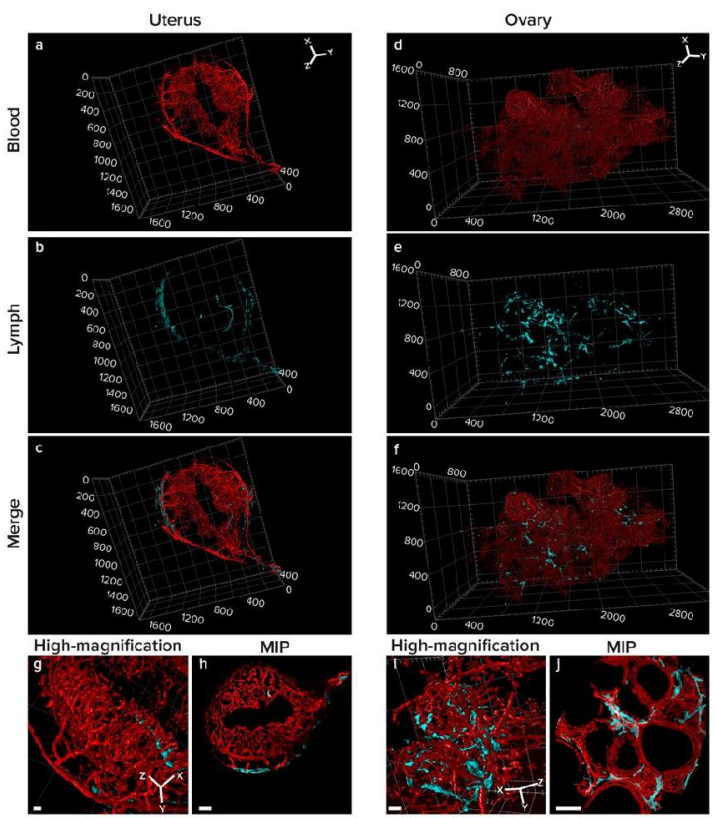

Figure 2 3-D reconstruction of blood and lymphatic vascular network of mouse uterus and ovary using WOBLI method. Blood vessels were in red channel and lymphatic vessles were in cyan channel. ${ }^{[27]}$ 


\section{Near-Infrared Fluorescence Imaging}

Near-infrared fluorescence (NIRF) imaging technique has been used to visualize lymphatic vasculature system in animals and human. Due to its reduced tissue absorption, small photon scattering and low auto fluorescence of biological tissues, NIRF imaging is a novel real-time non-invasive in vivo imaging technique with high resolution and tissue penetration. Naked eyes can directly see the lymphatic system with NIRF imaging system without any special treatment. Thus, NIRF imaging system is an emerging technology in clinical practice to visualize lymphatic vasculature system and diagnose the lymphatic physiological and pathological statement in several lymphatic-related diseases. $^{[28-30]}$

Indocyanine green (ICG), a dye that is approved in humans for intravenous administration, has been used in hepatic and cardiovascular testing and retinal angiography, and it can be excited by tissue-penetrating near-infrared light (the light with wavelength above $780 \mathrm{~nm}$ ). While poorly fluorescent, ICG may be used in humans in an off-label, intradermal route of administration for interrogation of the lymphatics. ${ }^{[31]}$ In recent years, researchers have improved the biodistribution properties of ICG in the in vivo lymphatic system through various techniques and has used NIRF imaging technology to perform in vivo imaging of lymphatic vessels and obtain the highly accurate anatomical, physiological and pathological properties of lymphatic vessels in vivo. All the results suggest that NIRF imaging technology based on ICG administration has good application prospects and clinical transformation value in lymphatic pathology research and diagnosis in lymphatic vessel related disease.

Nakajima et al. detected there previously known collecting lymphatic systems and two new lymphatic systems in the mouse hindlimb by administration ICG into foot pad, ankle, abdomen and lower back with the NIRF imaging system. After performing the lymphadenectomy of the popliteal, inguinal, and iliac lymph nodes in mice, researchers injected ICG and performing lymphangiography on day 58 and day 60 after lymphadenectomy in order to assess the lymph flow volume related with lymphedema in the hindlimb and then presumed the relationship between the lymphatic vasculature system and the lymphedema in the hindlimb. ${ }^{[32]}$

Kwon et al. used NIRF imaging techniques to image ICG distribution along the neuroaxis and the routes of ICG-laden cerebrospinal fluid outflow into the lymphatics following intrathecal lumbar administration. In this study, lymphatic contractile function and drainage pattern in peripheral lymph vessels and the in vivo dynamics and routes of cerebrospinal fluid outflow into the peripheral lymphatics were visualized through the administration of ICG. The results suggested that NIRF imaging could be used as a tool to probe CSF pathology including neurological disorders by imaging CSF outflow dynamics to lymphatics. ${ }^{[33]}$ Kwon et al. also investigated functional and architectural changes of inguinal-to-axillary internodal collecting lymphatic vessels (CLVs) in mice after a single axillary lymph node dissection using fluorescence imaging with intradermal injection of ICG and Alexa-680BSA to the paw and to the base of the tail. Through the fluorescence imaging, researchers found that lymph flow in the CLVs is affected by lymph node dissection and long-term interruption of lymph flow might cause CLV dysfunction and thus contribute to chronic lymphatic disorders. $^{[34]}$

Owing to the poor lymphatic distribution and retention of free ICG, Kraft et al. synthesized ICG nanoparticles composed by ICG and lipid excipients DSPC and DSPE-mPEG2000 and subcutaneous administrated those ICG in mice followed with NIRF imaging. The ICG nanoparticles provided selective lymphatic uptake, lymph vessel and node retention, and extensive first-pass lymphatic distribution of ICG, enabling $0.2 \mathrm{~mm}$ and 5-10 cell resolution of lymph vessels, and high signal-to-background ratios for lymphatic vessel and node networks (Figure 3). The properties of these particles may be used to diagnose as well as treat lymphatic abnormalities by enhancing drug exposure in lymph nodes and vessels. These particles may be also further developed to specifically interact with cell populations and signaling pathways in the lymphatic system to mediate immune responses. ${ }^{[35]}$

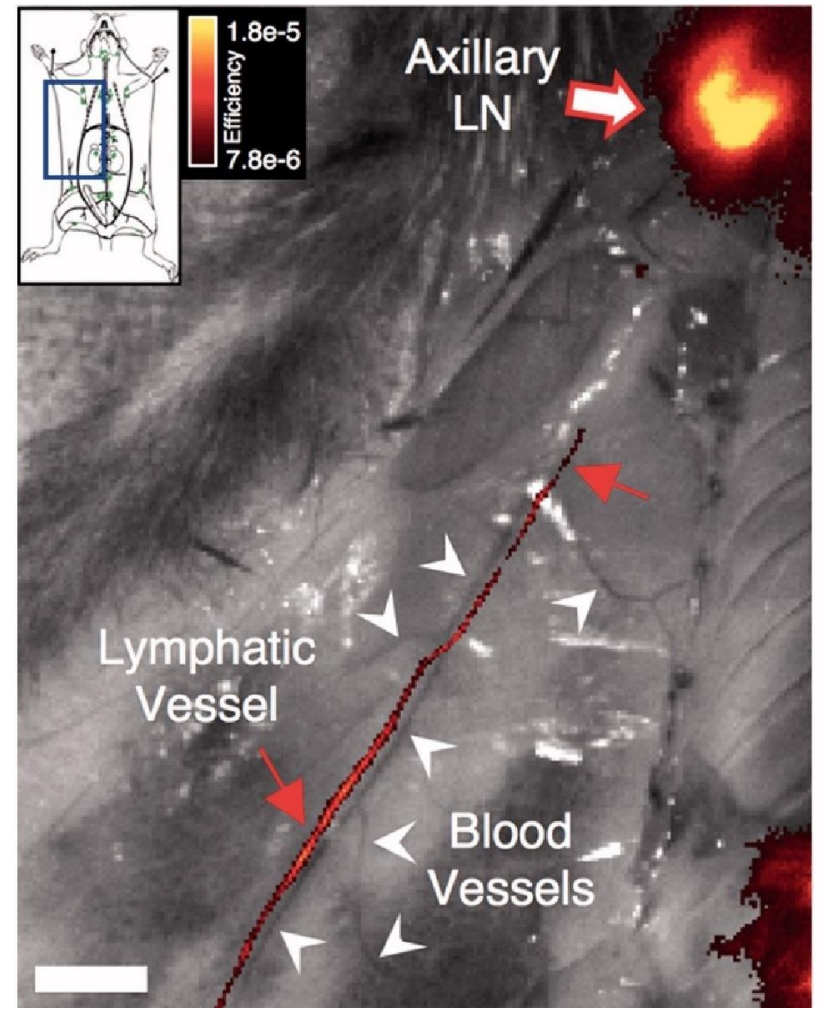

Figure 3 ICG nanoparticles detected a small lymphatic vessel (stemmed arrows) adjacent to blood vessels (non-stemmed arrows). The detected small lymphatic vessel connected the right sub iliac and axillary lymph nodes. Scale Bar, $3 \mathrm{~mm} .{ }^{[35]}$

Brambilla et al. manufactured soluble microneedles with the combination of poly( $N$-vinylpyrrolidone) and ICG to structurally and functionally assess the architecture, contractility and drainage quantification of dermal lymphatic system in vivo in mice by administrating the ICG microneedles and using a near-infrared camera. This painless functional "tattooing" procedure can be used for quantitative assessment of the dermal lymphatic function in several dermal conditions and treatment-response evaluations, staging several pathological conditions and evaluate the efficacy of drugs in several disease including lymphedema, skin cancer and skin chronic inflammation. ${ }^{[36]}$

\section{Photoacoustic Imaging}

Photoacoustic imaging is a novel real-time molecular imaging technique with high spatial resolution and great penetration depth inside the tissue, and photoacoustic lymphangiography is based on photoacoustic imaging. Because the reaction patterns of ICG draining into the lymphatic vessels and hemoglobin in blood vessels to the lasers are different, the use of two different laser wavelengths and photoacoustic imaging can differentiate lymphatic vessels from blood vessels in the superficial regions less than $20 \mathrm{~mm}$ in depth noninvasively with high resolution. ${ }^{[37-39]}$ 


\section{Minireview}

Kajita et al. performed photoacoustic lymphangiography after subcutaneous injection of ICG in a patient with a history of right axillary lymph node dissection for malignant lymphoma. Three-dimensional reconstruction of lymphatic vessels or both lymphatic and venous vessels (Figure 4) showed the complicated network of dilated lymphatic vessels and their relationship with the superficial veins of the patient's right forearm. ${ }^{[40]}$
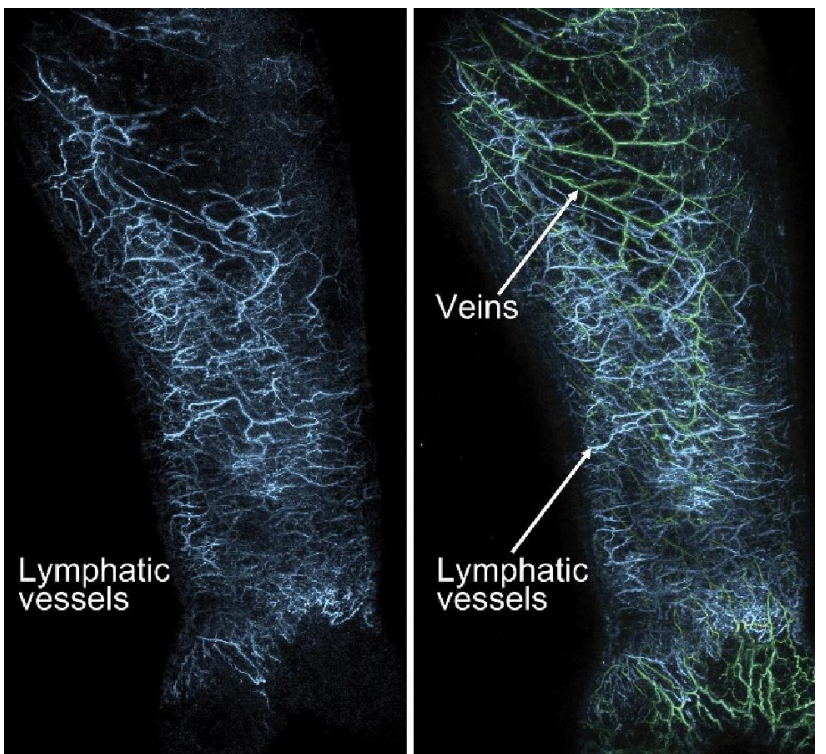

Figure 4 Images from photoacoustic lymphangiography of the forearm after subcutaneous injection of $0.5 \mathrm{~mL}$ ICG. Lymphatic vessels were in blue channel and superficial veins were in green channel. ${ }^{[40]}$

\section{Optical Coherence Tomography}

Optical coherence tomography (OCT) is an imaging technique which enables label-free 3-D imaging at micron-scale resolution and it can achieve angiography and lymphangiography, and in vivo OCT imaging is a non-invasive optical imaging technique. Compared with other optical microscope imaging techniques, OCT imaging technology of living subjects can improve the penetration depth of optical signals ${ }^{[41]}$ and provide 3-D images with high spatial resolution. ${ }^{\text {[42] }}$

As a pilot study of in vivo conjunctival OCT imaging, Gong et al. used OCT and optical coherence microscopy (OCM) to image lymphatic vessels in isolated conjunctiva of porcine eyes. In this study, the researchers segmented the lymphatic vasculature network by compensating the depth attenuation in the volumetric OCT/OCM signal, projecting the minimum intensity in a 2-D model and performing threshold processing to generate 3-D lymphatic volume information. The imaging method used in this study can perform label-free lymphangiography without tissue treatment and provide morphological information of lymphatic vessels for further study of lymphatic vessel structures in isolated conjunctiva and various tissues. This technique is also expected to be used in guiding and monitoring glaucoma filtration surgery. ${ }^{[43]}$

Si et al. used the OCT imaging technique to perform first living observation of the down-regulation of lymphatic specific biomarker LYVE-1 in the skin inflammation mouse model induced by acute contact hypersensitivity. In this study, the researchers used polystyrene-based microbeads ( $\mu \mathrm{Bs})$ as OCT contrast agents. In order to improve the binding ability of $\mu \mathrm{Bs}$ to LYVE-1 on lymphatic endothelial cells, researchers used streptavidin to coat $\mu \mathrm{Bs}$ and injected biotinylated antibodies against LYVE-1 before OCT imaging. According to the stronger binding ability of streptavidin to biotinylated antibody than that of antigen-antibody, the pre-targeting method mentioned above enables $\mu \mathrm{Bs}$ to specifically target LYVE-1 expressed on lymphatic endothelial cells and successfully performed OCT in vivo imaging based on phase variance algorithm. This study demonstrates the feasibility of in vivo molecular OCT imaging methods and lays the solid foundation for future clinical transformation studies of in vivo OCT imaging. ${ }^{[44]}$

\section{Conclusions and Perspectives}

The existing fluorescence imaging technologies have greatly promoted the understanding of the relationship between the morphological structure of lymphatic vasculature system and several lymphatic-related pathological conditions such as lymphedema and Crohn's disease. Based on the preliminary immunofluorescence techniques, researchers studied the status of lymphatic vessels in some pathological tissue and mouse models and gained more detailed morphological and functional information of lymphatic vessels including diameter, number and flow volume by using high-resolution microscopy imaging techniques such as confocal imaging, light sheet imaging and etc. The in vivo fluorescence imaging techniques especially the NIRF imaging technology based on fluorescent dye ICG have been applied maturely not only in scientific research of lymphatic vasculature system, but also in clinical application to determine the status of lymphatic vessels in patients. Novel in vivo fluorescent imaging technologies such as intravital OCT, photoacoustic lymphangiography and two-photon imaging are also driving the development of high spatial resolution in vivo fluorescence imaging of lymphatic vasculature system with brilliant clinical application prospect. However, we need to realize in vivo real-time high-resolution imaging of lymphatic vasculature system through combining with multi-modal multi-scale imaging technology. We still need to explore the structure and function of deep tissue (such as brain) lymphatic vasculature system with specific lymphatic probes and animal models. We also need establish the correlation between the signals presented by multi-modal and multiscale lymphatic imaging techniques and developing new methods for the application of molecular probes and molecular imaging techniques in the structural and functional imaging of lymphatic system.

\section{Acknowledgement}

This study was supported by grants from the National Key R\&D Program of China (No. 2016YFC1303100), the National Natural Science Foundation of China (Nos. 81671738, 81301261, and 21374059), the 13th Undergraduate Training Programs for Innovation of Shanghai Jiao Tong University School of Medicine.

\section{References}

[1] Mäkinen, T.; Jussila, L.; Veikkola, T.; Karpanen, T.; Kettunen, M. I.; Pulkkanen, K. J.; Kauppinen, R.; Jackson, D. G.; Kubo, H.; Nishikawa, S.; Ylä-Herttuala, S.; Alitalo, K. Nat. Med. 2001, 7, 199.

[2] Alitalo, K. Nat. Med. 2011, 17, 1371.

[3] Padera, T. P.; Meijer, E. F.; Munn L. L. Annu. Rev. Biomed. Eng. 2016, 18, 125.

[4] Skobe, M.; Hawighorst, T.; Jackson, D. G.; Prevo, R.; Janes, L.; Velasco, P.; Riccardi, L.; Alitalo, K.; Claffey, K.; Detmar, M. Nat Med. 2001, 7, 192.

[5] Peinado, H.; Zhang, H.; Matei, I. R.; Costa-Silva, B.; Hoshino, A.; Rodrigues, G.; Psaila, B.; Kaplan, R. N.; Bromberg, J. F.; Kang, Y.; Bissell, M. J.; Cox, T. R.; Giaccia, A. J.; Erler, J. T.; Hiratsuka, S.; Ghajar, C. M.; Lyden, D. Nat. Rev. Cancer 2017, 17, 302.

[6] Hirakawa, S.; Brown, L. F.; Kodama, S.; Paavonen, K.; Alitalo, K.; Detmar, M. Blood 2007, 109, 1010.

[7] Hoshida, T.; Isaka, N.; Hagendoorn, J.; Di Tomaso, E.; Chen, Y. L.; 
Pytowski, B.; Fukumura, D.; Padera, T. P.; Jain, R. K. Cancer Res. 2006, 66, 8065.

[8] Fernández-Suárez, M.; Ting, A. Y. Nat. Rev. Mol. Cell Biol. 2008, 9, 929.

[9] Ntziachristos, V.; Ripoll, J.; Wang, L. V.; Weissleder, R. Nat. Biotechnol. 2005, 23, 313

[10] Mcdonald, D. M.; Choyke, P. L. Nat. Med. 2003, 9, 713.

[11] Hernot, S.; van Manen, L.; Debie, P.; Mieog, J. S. D.; Vahrmeijer, A. L. Lancet Oncol. 2019, 20, 354.

[12] Zhang, R. R.; Schroeder, A. B.; Grudzinski, J. J.; Rosenthal, E. L.; Warram, J. M.; Pinchuk, A. N.; Eliceiri, K. W.; Kuo, J. S.; Weichert, J. P. Nat. Rev. Clin. Oncol. 2017, 14, 347.

[13] Van Leeuwen, F. W. B.; Winter, A.; Van Der Poel, H. G.; Eiber, M.; Suardi, N.; Graefen, M.; Wawroschek, F.; Maurer, T. Nat. Rev. Urol. 2019, 16, 159

[14] Banerji, S.; Ni, J.; Wang, S. X.; Clasper, S.; Su, J.; Tammi, R.; Jones, M.; Jackson, D. G. J. Biochem. Cell Biol. 1999, 144, 789

[15] Wigle, J. T.; Oliver, G. Cell 1999, 98, 769.

[16] Le Guen, L.; Karpanen, T.; Schulte, D.; Harris, N. C.; Koltowska, K.; Roukens, G.; Bower, N. I.; Van Impel, A.; Stacker, S. A.; Achen, M. G.; Schulte-Merker, S.; Hogan, B. M. Development 2014, 141, 1239.

[17] Kadota, K.; Huang, C. L.; Liu, D.; Nakashima, N.; Yokomise, H.; Ueno, M.; Haba, R. Lung Cancer 2010, 70, 88.

[18] Mermod, M.; Bongiovanni, M.; Petrova, T.; Goun, E.; Simon, C.; Tolstonog, G.; Monnier, Y. Otolaryngol. Head and Neck Surg. 2019, $160,277$.

[19] Feld, L.; Cifu, A. S.; Glick, L. R. J. Am. Med. Assoc. 2019, 321, 1822

[20] Pedica, F.; Ligorio, C.; Tonelli, P.; Bartolini, S.; Baccarini, P. Virchows Arch. 2008, 452, 57.

[21] D'Alessio, S.; Correale, C.; Tacconi, C.; Gandelli, A.; Pietrogrande, G.; Vetrano, S.; Genua, M.; Arena, V.; Spinelli, A.; Peyrin-Biroulet, L.; Fiocchi, C.; Danese, S. J. Clin. Invest. 2014, 124, 3863.

[22] Rehal, S.; Von Der Weid, P. Y. Am. J. Pathol. 2017, 187, 798.

[23] Randolph, G. J.; Bala, S.; Rahier, J. F.; Johnson, M. W.; Wang, P. L.; Nalbantoglu, I.; Dubuquoy, L.; Chau, A.; Pariente, B.; Kartheuser, A.; Zinselmeyer, B. H.; Colombel, J. F. Am. J. Pathol. 2016, 186, 3066.

[24] Lohrberg, M.; Wilting, J. Cell Tissue Res. 2016, 366, 667.

[25] Nizamutdinova, I. T.; Maejima, D.; Nagai, T.; Meininger, C. J.; Gashev, A. A. Lymphatic Res. Biol. 2017, 15, 136.

[26] Oren, R.; Fellus-Alyagor, L.; Addadi, Y.; Bochner, F.; Gutman, H.;
Blumenreich, S.; Dafni, H.; Dekel, N.; Neeman, M.; Lazar, S. Sci. Rep. 2018, 8, 1.

[27] Park, C.; Hwang, I. Y.; Kehrl, J. H. Methods Mol. Biol. 2016, 1407, 195.

[28] Hong, G.; Antaris, A. L.; Dai, H. Nat. Biomed. Eng. 2017, 1, 0010.

[29] Vahrmeijer, A. L.; Hutteman, M.; Van Der Vorst, J. R.; Van De Velde, C. J. H.; Frangioni, J. V. Nat. Rev. Clin. Oncol. 2013, 10, 507.

[30] Chinnathambi, S.; Shirahata, N. Sci. Technol. Adv. Mater. 2019, 20 , 337.

[31] Sevick-Muraca, E. M.; Kwon, S.; Rasmussen, J. C. J. Clin. Invest. 2014, 124, 905.

[32] Nakajima, Y.; Asano, K.; Mukai, K.; Urai, T.; Okuwa, M.; Sugama, J.; Nakatani, T. Sci. Rep. 2018, 8, 7078.

[33] Kwon, S.; Janssen, C. F.; Velasquez, F. C.; Sevick-Muraca, E. M. J. Immunol. Methods 2017, 449, 37.

[34] Kwon, S.; Price, R. E. Biomed. Opt. Express 2016, 7, 1100.

[35] Kraft, J. C.; Treuting, P. M.; Ho, R. J. Y. J. Drug Target. 2018, 26, 494.

[36] Brambilla, D.; Proulx, S. T.; Marschalkova, P.; Detmar, M.; Leroux, J. C. Small 2016, 12, 1053.

[37] Omar, M.; Aguirre, J.; Ntziachristos, V. Nat. Biomed. Eng. 2019, 3, 354.

[38] Nagae, K.; Asao, Y.; Sudo, Y.; Murayama, N.; Tanaka, Y.; Ohira, K.; Ishida, Y.; Otsuka, A.; Matsumoto, Y.; Saito, S.; Furu, M.; Murata, K.; Sekiguchi, H.; Kataoka, M.; Yoshikawa, A.; Ishii, T.; Togashi, K.; Shiina, T.; Kabashima, K.; Toi, M.; Yagi, T. F1000Research 2019, 7, 1813.

[39] Zackrisson, S.; Van De Ven, S. M. W. Y.; Gambhir, S. S. Cancer Res. 2014, 74, 979.

[40] Kajita, H.; Kishi, K. Radiology 2019, 190241.

[41] Huang, D.; Swanson, E. A.; Lin, C. P.; Schuman, J. S.; Stinson, W. G.; Chang, W.; Hee, M. R.; Flotte, T.; Gregory, K.; Puliafito, C. A.; Fujimoto, J. G. Science 1991, 254, 1178.

[42] Wojtkowski, M. Appl. Opt. 2010, 49, D30.

[43] Gong, P.; Yu, D. Y.; Wang, Q.; Yu, P. K.; Karnowski, K.; Heisler, M.; Francke, A.; An, D.; Sarunic, M. V.; Sampson, D. D. J. Biophotonics 2018, 11.

[44] Si, P.; Sen, D.; Dutta, R.; Yousefi, S.; Dalal, R.; Winetraub, Y.; Liba, O.; de La Zerda, A. Sci. Rep. 2017, 7, 1086.

Received July 21, 2019 Accepted August 6, 2019 